\title{
Research on the Routing Protocol Analysis in Wireless Ad Hoc Networks LIU Yang ${ }^{1, a}$ \\ ${ }^{1}$ Chongqing College Of Electronic Engineering, Chongqing 401331, China \\ aliuyang023023@sina.com
}

Keywords: Routing Protocol, Analysis, Wireless MANET

\begin{abstract}
Wireless MANETs with the features of flexible, high overall robustness and low system costs and it has attracted wide attention. In wireless ad hoc networks, routing protocol occupies an important position. The paper analyzes the routing protocol in wireless ad hoc network to achieve high-performance routing protocol.
\end{abstract}

\section{Introduction}

Wireless ad hoc network (Ad Hoc) nodes can dynamically join and leave the network, the networking flexibility, robustness of the network as a whole high and low system cost advantages make the Ad Hoc network has an irreplaceable role and broad application and development prospects.

Ad Hoc network itself particularity and importance of routing protocols, dynamic routing topology requirements must be established promptly, limited wireless network resources requires routing protocol must have smaller overhead and energy consumption. Traditional routing technology has been unable to adapt to the dynamic changes of the Ad Hoc network topology, we must design new features for Ad Hoc network routing protocols. At present, many domestic and foreign universities and research-related institutions have begun Ad Hoc Ad Hoc network in particular Research on routing technology. In the late nineties of the last century, a number of research institutions to submit to the MANET routing protocol working group, such as a dynamic source routing Carnegie Malone University submitted DSR, C-K and Toh submitted ABR. These routing protocols each based on different starting point and measure, through the mechanism needed to solve the dynamic problems caused by topology.

\section{The Development of Wireless Ad Hoc Networks}

Origin of wireless ad hoc networks can be traced back to 1968, when, and educational institutions in order to exchange data with the Hawaiian Islands, the United States established a radio technology using packet switched computer network (ALOHA network), although initially only a single hop network protocols, but ALOHA network uses wireless packet switching technology is the future for the development of wireless ad hoc network foundation. In recent years, wireless ad hoc networks in the military sphere gradually practical. The US military tactics implemented in 1997 the Internet is currently the largest multi-hop packet radio network. Although early MANETs development is primarily driven by military needs, but its use in civilian areas has also been considerable progress. To the 1990s, based on the emergence of a variety of radio frequency and infrared wireless communication devices as well as popular laptops, making wireless ad hoc network in the field of civilian communications have a huge application space. Currently, wireless ad hoc networks a universal concern. Based on some of the International Organization for Standardization wireless networking technology, the formation of a series of standards and the draft on the physical layer, data link layer and the network layer [1].

\section{The Characteristics of Wireless Ad Hoc Networks}

Wireless ad hoc network is widely used, compared with traditional communication networks, wireless ad hoc network has the following characteristics: 
No Center and Self-Organization. Status of wireless ad hoc network nodes are equal, there is no strict control center, is a peer to peer network nodes can join or leave the network at any time. And cable networks, the biggest difference between the Ad Hoc network is that you can at any time, any place to quickly build up a mobile communications network, without the need for network infrastructure hardware architecture. Features flexible Ad Hoc network is suitable for disaster relief, communications and other applications in remote areas.

Dynamic Change of Network Topology. As a dynamic network, wireless ad hoc network hosts can move anywhere, you can join or leave at any time, which will lead to changes in network topology, and changes in the way and speed are hard to predict. The conventional network topology is relatively constant [2].

Energy Limitations. In wireless ad hoc networks, terminals are some mobile devices, such as a PDA, a portable computer or PDA. Since the terminal may be in a state under constantly moving, and without wired infrastructure, energy, mainly provided by the host cell, so the wireless ad hoc networks have limited energy characteristics.

The Distributed Nature of the Network. Equal node in wireless ad hoc networks, distributed protocol host connectivity. One or some of the nodes of the network fail and the remaining node can still work properly. Wireless ad hoc network communication terminal along with routing and host functions, communication protocols and routing algorithms used in the network usually distributed control mode, thus invulnerability than centralized control of the network stronger.

Communication Bandwidth Is Limited. Wireless ad hoc network does not support a wired infrastructure, communication between nodes through the wireless channel, and wireless channel bandwidth is itself provides much lower than the cable network, which is determined by the physical characteristics of the decision. In addition, since the wireless signal has path attenuation, and are subject to signal interference and noise impact, the state of the wireless link is very easy to change, the link is far better than the actual effective throughput of the calculated maximum theoretical bandwidth.

Limited Physical Security. Wireless networks are typically more vulnerable to physical security attacks than wired networks, vulnerable to eavesdropping, deception and denial of service attacks. Existing link security techniques have been applied in some wireless networks. However, the distributed nature of wireless ad hoc networks has to make it relative to the centralized network, with better survivability.

The Life Cycle Is Short. Wireless ad hoc network is mainly used to meet the communication needs temporary respect the wired network, its survival time is usually shorter.

Dynamically Allocate Resources. Including radio channel, the channel rate, power, routing and other network resources, can be dynamically allocated by the algorithm, on-demand services.

\section{The Routing Protocol in Wireless Ad Hoc Network}

A good routing algorithms to consider not only the limited network resources, dynamically changing network topology, improving network throughput and other aspects of factors, but also consider the entire network fault tolerance. Wireless ad hoc network routing scheme has its particularity, there are differences with the traditional routing schemes, mainly in the following points [3].

The traditional routing scheme relies on a network terminal or saved in some specific management terminal in a distributed routing database, and for mobile wireless ad hoc networks, terminals cannot move freely, and may at any time on and off, which will make flexible network topology. Thus, the mobile terminal cannot be permanently stored routing information, and routing information is also stored in them is not always reliable. Different routing wireless ad hoc networks and fixed network also lies in the packet forwarding wireless ad hoc network is completed by a common network terminal, rather than done by a dedicated router;

The existing of unidirectional channel. In a traditional network routing protocol, the transmission channel is generally between nodes is bidirectional, but in the use of wireless communications from networking environments, due transmit power or geographical location and other factors, may cause 
a one-way channel.

Due to the physical characteristics of the radio channel itself, the network bandwidth it provides relatively limited channel is much lower. Moreover, taking into account the impact of competition arising shared radio channel and signal attenuation, noise variety of factors, inter-channel interference, the actual bandwidth of the results obtained is far less than the theoretical maximum bandwidth value [4].

\section{The Research on Single-Path Routing and Multi-Path Routing}

From the routing path points, the current Ad Hoc network by communication model can be divided into single-path routing and multi-path consists of two categories, the following were to be introduced [5].

Single-Path Routing. The traditional classification methods divided Ad Hoc network routing into "table-driven" and "demand-driven" two categories, table-driven routing and the network of any one node to all other nodes to maintain a routing table. When the network topology changes, the between nodes updates this information to maintain the correct routing table. Demand-driven routing, and only when there is routing the source node initiates a route discovery needs to find the right path for a particular destination node in the network. In using this path, the network node to maintain relevant and effective messages routed through interaction and remove the stale routes between multipath routing refers to the source / destination communication node to establish multiple paths do not intersect at the same time in the same grouped delivery, multiple paths to the source node and the destination node can compensate for dynamic characteristics between mobile Ad Hoc networks and unpredictability, thus improving the communication quality of the communication between the two sides service.

This traditional classification approach is too vague, routing protocols can also be divided into flat and hierarchical routing protocol routing protocol based on the network structure of Ad Hoc. Plane routing protocol does not rank in the network division, all network nodes have the same function as the priority, they receive the same motion routing control information. The hierarchical routing protocol routing needs to distinguish between different levels when processing nodes information in order to reduce the number of control packets. Hierarchical routing protocol is divided into two types, one called neighbor selection, the agreement node dynamically routed according to its neighbor nodes; the other is called the partition selection and that is based on network topology management division range.

Routing protocols can be divided into routing protocols based on the destination node topology and state information based on the Ad Hoc network. Need to save a large number of topology information nodes topology-based routing protocol, which is the principle of link-state protocol is the same. Routing protocol is based on the destination node in the node does not need to save a lot of topology information, only the information needed to save the topology of the nearest node, the most famous of such agreements is a distance vector routing protocol, which requires maintenance to the destination node distance vector .

In addition, according to the routing can be divided into first should establish routing protocol and passive routing protocols. Proactive routing protocols to table-driven routing protocol is represented by each network node to the destination node stored routing information, all of the nodes periodically exchange routing information. Passive routing protocol process can be divided into routing path discovery and maintained path found only when the source node will not start until there is a demand.

Multi-Path Routing. Multiple path transport is the use of multiple paths to deliver applications disjoint grouping mechanism in order to increase the bandwidth and reliability of connection. Mobile Ad hoc networks, due to lack of infrastructure, network topology changes dynamically, its support for QoS weak. But its structure and the user's mobile network increases flexibility, diversity of users and network capacity of the network, which are provided for in which the implementation of the MPT favorable factors.

Multipath routing model is any pair of nodes simultaneously provides multiple paths available, 
and allows nodes to a host (or application) to choose how to use these paths. Multipath routing algorithm provides multiple paths between nodes and ensures that data sent to a path which reaches their destinations via the path. To calculate the path between nodes, the path must be specified characteristics depending on the application path. As of end to end in order to get the maximum throughput, the path must be specified characteristics are: any node in the aggregate throughput for multiple paths between the maximum. In order to obtain the minimum end to end delay, it is very necessary to predetermined characteristics of the path is: at any time, there is at least one path having a minimum delay between any pair of nodes. Path specification defines the characteristics of the particular set of paths and the path computation algorithm to calculate the path set in line with the actual path specifications defined features.

Path type specifies the relationship between multiple paths between nodes. There are two types of paths: multi-path multi-service and multi-path multi-choice. Multipath is a multi-service multiple paths having different characteristics. For example, the network can provide both high bandwidth and low latency path, which allows applications to choose the path that best meet their communication requirements. For example, the network may provide four paths to high-bandwidth services that each node has four high-bandwidth paths to any destination node. But in general multi-service multi-path selection algorithm generally more complex and difficult to achieve, and multi-path multi-choice selection algorithm is relatively easy, it is now more commonly used multi-path routing multiple choice.

\section{Conclusions}

The routing protocol in wireless ad hoc network is the research focus in this field, in which the energy validity of the agreement is the major problem that troubled the technological development and the dynamic topology in wireless ad hoc network is the greatest difficulty in routing processing. The current various routing protocols in wired network cannot meet the needs and it must develop new routing protocols applied to the wireless ad hoc network. In routing protocol design, we need to consider the distribution, efficiency, timeliness, safety, and energy efficiency and other issues of the algorithm.

\section{References}

[1] Lijie Wu: Microelectronics \& Computer, Vol. 10 (2006) No 26, p.219-222

[2] Weifeng Zhai: Computer Engineering, Vol. 12 (2005) No 27, p.74-76

[3] Xiaomei Wang: Computer Engineering, Vol. 1 (2006) No 33, p.11-14

[4] Minqiang Wang: Nanjing Posts Institute, Vol. 1 (2005) No25, p.13-17

[5] Li Xu: Computer Systems, Vol. 2 (2006) No27, p.212-215 